\title{
Effects of a specific task-oriented training versus its combination with manual therapy on balance and mobility in people post stroke at the chronic stage: study protocol for a pilot randomised controlled trial
}

\section{Kristina Traxler}

Therapiezentrum Kinema

\section{Franz Schinabeck}

Überörtliche Gemeinschaftspraxis Hohenwarth/Lam

\section{Eva Baum}

Therapiezentrum Kinema

\section{Edith Klotz}

Praxis Mittelpunkt Mensch

Barbara Seebacher ( $\nabla$ barbara.seebacher@i-med.ac.at)

Medical University of Innsbruck: Medizinische Universitat Innsbruck https://orcid.org/0000-0001-56999077

\section{Study Protocol}

Keywords: Stroke, Neurological physiotherapy, Manual therapy, Task-oriented training, Postural balance, Walking, Mobility limitation, Range of motion, Ankle joint.

Posted Date: December 23rd, 2020

DOl: https://doi.org/10.21203/rs.3.rs-132414/v1

License: (9) This work is licensed under a Creative Commons Attribution 4.0 International License. Read Full License

Version of Record: A version of this preprint was published at Pilot and Feasibility Studies on July 27th, 2021. See the published version at https://doi.org/10.1186/s40814-021-00886-0. 


\section{Abstract}

Background: Large studies have shown that stroke is among the most relevant causes of acquired adult disability. Walking and balance impairment in stroke survivors often contribute to a restriction in daily activities and social participation. Task-oriented training (TOT) is an effective treatment strategy and manual therapy (MT) is used successfully to enhance ankle joint flexibility in this population. No study, however, has compared TOT against its combination with MT in a randomised controlled trial. Aims of this pilot study are therefore to explore the feasibility of a full-scale RCT and the preliminary effects of specific TOT with a combined specific TOT-MT versus a control group in people post stroke.

Methods: This is a protocol of a 4-week prospective randomised controlled parallel pilot trial in people post stroke at the chronic stage with limited upper ankle joint mobility and an impairment in balance and mobility. At a German outpatient therapy centre using 1:1:1 allocation, 36 patients will be randomised into one of three groups: 15-minute talocrural joint MT plus 30-minute specific TOT (Group A); 45-minute specific TOT (Group B); controls (Group C). Training will be goal-oriented including tasks that are based on daily activities and increased in difficulty ulilising predefined progression criteria. Interventions will be provided face-to-face 2 times per week, for 4 weeks, in addition to 20-minute concurrent $x 4$ weekly homebased training sessions. Data will be collected by blinded assessors at baseline, post-intervention and 4-week follow-up. The primary outcome will be feasibility assessed by recruitment, retention and adherence rates, compliance, adverse events, falls and the acceptability of the intervention. Secondary outcomes will be walking speed, single and dual tasking functional mobility, ankle range of motion, disability and health-related quality of life.

Discussion: Results from this study will inform a larger randomised controlled trial to investigate the effects of specific TOT and specific TOT-MT compared to a post stroke control group. We hope that the study findings help to enhance stroke rehabilitation.

Trial registration: German Clinical Trials Register, DRKS00023068. Registered on 21.09.2020, https://www.drks.de/drks_web/navigate.do?navigationld=trial.HTML\&TRIAL_ID=DRKS00023068.

\section{Introduction}

Walking and balance impairment contribute to functional disability experienced by many stroke survivors, frequently affecting social participation (1). Postural control deficits in the stroke population are related to reduced walking speed and dynamic mobility, greater gait variability and fall rates (2). Functional walking limitation and motor control deficits in people post stroke are associated with the upper motor neuron syndrome (3). Foot drop is often observed in stroke patients, induced by spasticity, weakness of the foot dorsiflexors, shortening of plantarflexors and ankle range of motion (ROM) limitation $(4,5)$. Such a complex movement disorder requires differentiated therapy strategies, to address both the structural and functional movement limitations. Upper ankle joint i.e., talocrural manual therapy (MT) (6) and MT with movement leads to increased joint ROM alongside balance improvements $(7,8)$. 
Few studies have investigated the effects of talocrural mobilisation and conventional functional training in chronic stroke patients, showing conflicting results. Kluding and Santos compared the effects of functional practice with ankle joint mobilisations against functional practice alone (9). Study results showed increased ROM of the ankle joint after MT. Improved weight transfer to the paretic side during sitto-stand was seen after functional training whereas combined practice impaired weight transfer. An and Jo explored active talocrural mobilisation as an add-on to conventional physiotherapy in chronic stroke patients. In contrast to the aforementioned study, improvements in ankle ROM, strength, mobility and weight transfer to the paretic side were observed post-intervention (10).

From a neurological physiotherapy and motor learning perspective, it appears relevant practising meaningful tasks to enhance motor control and dynamic mobility. Specific strategies are needed to promote motor learning and long-term improvement in motor function (11). Task-oriented training (TOT) is established in stroke rehabilitation, including a variety of trained activities and progression strategies $(12-14)$ where patients actively interact with the environment striving for a defined and meaningful goal (15). While the training is individually targeted, progression should be predefined. Patients will be invited to choose training tasks according to their preference and activities of daily living (ADL). In addition, effective training programmes involve a specific, outstanding, challenging, repetitive, variable and intensive task practice (16-18). It appears logical that patients who consider the training programme useful and designed for their daily needs engage more intensively. Finally, significant motor learning literature has suggested adopting an external focus of attention during the training, directed to the desired outcome, a device or the environment (19). Conventional physiotherapy may not meet this criterion without explicit intention.

To our knowledge, only one study has investigated the effects of a specific TOT as compared to a combined wrist joint MT and upper-limb TOT approach (20). Hand function and joint mobility significantly improved after the combined training than compared to TOT alone. This cross-sectional study did not assess long-term outcomes. A randomised controlled trial (RCT) with a follow-up period is therefore needed which applies the combined intervention protocol to the lower limbs. This pilot study will be conducted to evaluate the feasibility of the methods, of a larger trial and to explore the preliminary effects of a specific TOT, versus its combination with talocrural mobilisation on balance, mobility, ankle ROM, number of falls and health-related quality of life (HRQoL) in people post stroke.

\section{Objectives}

Explore the feasibility of a larger RCT that will validate the study results and investigate the effects of specific task-oriented mobility and balance training versus its combination with MT on dynamic balance in people at the chronic post stroke stage. Secondary Objectives will be to investigate the preliminary effects the same training on dynamic balance, mobility, walking speed, upper ankle ROM, number of falls, disability and HRQoL.

\section{Study design and location}


The supporting CONSORT checklist for this study is available as additional information; see Additional file 1. This will be a prospective assessor-blinded randomised controlled exploratory single-centre pilot trial in chronic stroke patients comprising two parallel intervention groups and a primary outcome of feasibility of a full-scale RCT. Block randomisation will use permuted blocks of 3 and 6 and 1:1:1 allocation. A CONSORT flow diagram is shown in Fig. 1. The study location will be a physiotherapy practice in rural Germany, chosen because the homebased intervention has been designed for stroke patients at the chronic stage who have regained at least a minimum level of mobility.

\section{Methods}

\section{Participants}

\section{Inclusion Criteria}

Patients eligible for the trial must comply with all of the following at randomisation:

1. 18 to 98 years of age

2. People who have had an ischaemic or haemorraghic stroke at least 6 months prior to study start

3. Limited dorsiflexion ROM in the upper ankle joint on the hemiparetic side: maximum $0^{\circ}$ active ROM; maximum $5^{\circ}$ of passive ROM

4. Capability to independently transfer from a seating to a standing position without an assistive device - use of the hands is allowed

5. Ability to independently walk without an assistive device for at least 10 metres

6. Confirmation by the principle investigator $(\mathrm{PI})$ of the study (medical clinician) that there are no contraindications connected to exposure to active training

\section{Exclusion Criteria}

Patients will be excluded from the study if they meet any of the following criteria:

1. Any contraindication to MT (ankle instability, ankle inflammation or traumas etc.)

2. Any not fully recovered injuries of the musculoskeletal system

3. Previous surgery in the lower extremity region leading to walking impairment due to orthopaedic reasons

4. Relevant ROM reduction of the hip or knee joints

5. Any cognitive or communication impairment hindering participation in this pilot study according to the study PI (dementia, severe aphasia, being under legal guardianship etc.)

6. Diabetes mellitus

7. Rheumatic diseases

8. Chronic pain treated by drug therapy 
9. History of previous stroke with persistent, impairing neurological symptoms (defined by a Modified Rankin Scale score (mRS) (21) of $\geq 3$ )

10. Comorbidity hindering participation, according to the study PI (life expectancy $<12$ months, cancer, severe heart insufficiency etc.)

11. $\mathrm{mRS}$ score $\geq 4$

12. Pregnancy

13. Military service

\section{Recruitment and informed consent}

The study will be advertised using recruitment flyers at medical and physiotherapy practices within a $30 \mathrm{~km}$ radius of the study location, written informed consent being taken by a medical doctor as per German legislation. Additionally, a trained physiotherapist who holds a Master's degree in musculoskeletal physiotherapy and is studying for another Master's degree in neurological physiotherapy (KT) will explain the study intervention in detail. Oral and written information will be given in comprehensible lay language and questions answered regarding study procedures.

\section{Randomisation, concealment of allocation and blinding}

Randomisation will be stratified on age, the most relevant predictive factor for balance and mobility change in people post stroke (strata: under 70; 70 and over). Stratification will help ensure similarity between the intervention and control groups regarding age. A stratified block randomisation in permuted blocks of three or six with a random number generator will be performed (Sealed Envelope, London, UK). with details of blocking kept in a separate, unavailable document.

Allocation concealment will be performed to avoid allocation bias. Each participant will receive a unique identification number (ID). Based on the randomisation list, sequentially numbered sealed opaque envelopes including group allocation numbers $A, B$ and $C$ will be created for each stratum, given to participants in the order they are recruited. Participants will be asked not to discuss their allocation until study completion. The allocation sequence and sealed envelopes will be created by a researcher who is not involved in the study procedures (BS). Participants will be assessed for eligibility and enrolled by the chief investigator (FS) and assigned to the intervention or control groups by the care provider, a physiotherapist-researcher (KT). Outcome assessors (EB; EK) will be blinded to the group allocation of participants. It will not be feasible to blind the care provider. Participants will be blinded to the study hypotheses.

\section{Interventions}

Eligible patients will be randomised into one of three groups:

- Participants in Group A (Gp A) will receive a 15-minute talocrural joint MT directed at the dorsiflexion before a 30-minute specific task-oriented mobility and balance training 2 times per week. Further, a 20-minute home-based ankle mobilisation and TOT programme 4 times per week, for 4 weeks. 
- Participants in Group B (Gp B) will receive a 45-minute specific task-oriented mobility and balance training 2 times per week. Further, a 20-minute homebased TOT programme 4 times per week, for 4 weeks.

- Participants in Group C (Gp C) will receive no specific treatment.

All participants will receive their usual care. Interventions in groups A and B will be provided face-to-face by an experienced physiotherapist (KT). Participants will be asked to perform the homebased training on the intervention-free days only and given free choice of one rest day per week.

\section{Manual Therapy}

MT will be directed towards the upper ankle joint structures and aim to increase the dorsiflexion ROM. Two different talocrural mobilisation techniques will be used as shown in Fig. 2. Manipulation Terminology is described according to Mintken et al. (22).

\section{Face-to-face task-oriented training}

TOT will focus on activities involving mobility and dynamic balance. Activities will be based on the requirements of daily living, i.e avoiding or stepping over obstacles or picking-up objects from the ground. Participants will be timed while being instructed to perform the goal-oriented activities with as much effort and speed as possible. The number of repetitions (or objects moved) will be counted aloud by the physiotherapist researcher as motivation. Additionally, participants will be encouraged to put maximum effort into the TOT, while remaining comfortable. According to established principles of motor learning, all exercises should progress in difficulty. Moreover, participants can choose the task order from a predefined list. Between tasks, participants may rest for 45-60 seconds. Table 1 presents an intervention chart based on the template for intervention description and replication (TIDieR) checklist (23). Detailed information on the face-to-face and homebased intervention is provided in Additional File 2. 
Table 1

Intervention chart

\section{ITEM NO ITEM DESCRIPTION}

\begin{tabular}{llll}
\hline 1 BRIEF NAME & Group A & Group B & Group C \\
\cline { 2 - 4 } & $\begin{array}{l}\text { Upper ankle joint manual therapy (MT) } \\
\begin{array}{l}\text { \& task-oriented mobility and balance } \\
\text { training (TOT) }\end{array}\end{array}$ & $\begin{array}{l}\text { Task-oriented } \\
\text { mobility and balance } \\
\text { training (TOT) }\end{array}$ & $\begin{array}{l}\text { Control } \\
\text { group: no } \\
\text { intervention }\end{array}$
\end{tabular}

2 WHY

To evaluate the feasibility of the methods, of a larger trial and explore the preliminary effects of a specific TOT versus its combination with talocrural MT on balance, mobility, ankle range of motion (ROM), number of falls and healthrelated quality of life in people post stroke.

\section{WHAT MATERIALS}

- Information sheet on study procedures

- Information booklet on homebased TOT programme

- Exercise diary including a compliance checklist

\section{WHAT PROCEDURES}

- Usual care

- TOT introduction: in lay language; description of the principles

- Supervised TOT performance

- Homebased TOT

- MT introduction: in lay language;

description of the principles

- MT performance of the talocrural

joint

- Homebased ankle mobilising exercise

5 WHO PROVIDES
The intervention will be provided by a trained physiotherapist who holds a Master's degree in musculoskeletal physiotherapy and is studying for another Master's degree in neurological physiotherapy

6 HOW

- TOT and MT introduction and performance: individually and depending on the group participants will be allocated to

- Homebased training: individually

7 WHERE

- TOT and MT introduction and performance: at Outpatient therapy centre „Kinema", Germany

- Homebased training: At participants' homes

17 minutes, 6 times a week, for 4 weeks

8 WHEN AND HOW MUCH
15 min MT plus 30 min TOT, 2 times per week, for 4 weeks
45 min TOT, 2 times

per week, for 4 weeks 


\begin{tabular}{|c|c|c|c|}
\hline ITEM NO & \multicolumn{3}{|l|}{ ITEM DESCRIPTION } \\
\hline & \multicolumn{3}{|c|}{$\begin{array}{l}20 \text { min homebased training, } 4 \text { times per week, for } 4 \text { weeks; on } \\
\text { the supervised intervention-free days }\end{array}$} \\
\hline 9 TAILORING & Same intervention for all participants & $\begin{array}{l}\text { Same intervention for } \\
\text { all participants }\end{array}$ & $\begin{array}{l}\text { No } \\
\text { intervention }\end{array}$ \\
\hline $\begin{array}{l}10 \\
\text { MODIFICATIONS }\end{array}$ & No modifications & No modifications & $\begin{array}{l}\text { No } \\
\text { modifications }\end{array}$ \\
\hline \multirow[t]{3}{*}{$\begin{array}{l}11 \text { HOW WELL } \\
\text { PLANNED }\end{array}$} & \multicolumn{3}{|c|}{$\begin{array}{l}\text { - Intervention adherence and compliance will be assessed using a participant } \\
\text { diary, during appointments and at post-intervention }\end{array}$} \\
\hline & \multicolumn{3}{|c|}{$\begin{array}{l}\text { - Recording in excel sheets will be performed by the instructing researcher } \\
\text { (physiotherapist) }\end{array}$} \\
\hline & \multicolumn{3}{|c|}{ - The process will be supervised by the academic supervisor. } \\
\hline \multirow[t]{2}{*}{$\begin{array}{l}12 \text { HOW WELL } \\
\text { ACTUAL }\end{array}$} & \multicolumn{3}{|c|}{$\begin{array}{l}\text { - Adherence rates will be reported as median (range) times per week and } \\
\text { percentage ( } 95 \% \text { confidence interval): face-to-face and homebased training }\end{array}$} \\
\hline & \multicolumn{3}{|c|}{$\begin{array}{l}\text { - Compliance will be presented narratively (exercise types and order; number of } \\
\text { repetitions; problems and deviations from protocol with reasons or explanations) }\end{array}$} \\
\hline
\end{tabular}

\section{Homebased training}

The same homebased TOT programme will be used for participants in groups A and B. Participants will be informed they may select the TOT type and order but should alternate tasks over the intervention period. Tasks should be performed in sets of 3, E.g, (Task 2, Task 5, Task 7 and Task 8 ) 3 . Gp A will receive a self-mobilisation technique of the upper ankle joint. Participants will be instructed to commence their training with self-mobilisation. Participants in Gp B will perform another task-oriented exercise. Participants will be instructed to record their training in an exercise diary e.g., number of repetitions/time period. Similar to face-to-face training, training will progress in difficulty. A 1-minute pause will be scheduled between the various tasks, but not between sets of the same task (Additional File 2).

\section{Strategies to improve adherence to interventions}

A 4-week goal-oriented face-to-face intervention will be used. Goal setting and supervision of exercises by a physiotherapist have been shown to facilitate adherence (24). The study intervention will be based on ADL relevant to people post stroke which may enhance adherence. In addition, the described TOT could be motivating for study participants. The 1:1 treatment sessions will be scheduled at the patients' convenience.

As for home-based training, simple exercise instructions written in lay language and including photos will be employed to guide and improve training adherence. Participants will be allowed to freely choose the time, task order and type, however all tasks should be trained. Tasks will be demonstrated and explained by the physiotherapist. The scheduled duration of the home-based training is 20 minutes, likely perceived doable by participants. An exercise diary with a compliance checklist will be used to monitor adherence 
and checked by the physiotherapist weekly throughout the intervention period. as will adherence to the twice weekly sessions. Participants will be reminded of their post-intervention and follow-up appointments.

\section{Data collection}

Demographic (gender, age) and stroke specific data (lesion side; disease duration; modified Rankin scale score) will be extracted from patients' charts at eligibility screening $\left(-t_{1}\right)$. Study specific assessment data will be collected at baseline $\left(t_{1}\right)$, post-intervention $\left(t_{2}\right)$ and follow-up $\left(t_{3}\right)$ at 4 weeks after completion (Table 3) by two physiotherapists blinded to the study intervention. Assessments will be performed at similar times to increase comparability. In addition to ROM measurement, the validated German version of all assessments will be used. Semi-structured interviews will gather in-depth information regarding acceptance of the study intervention. Changes from baseline and from post-intervention will be evaluated for all outcomes using means, medians, proportions and text as appropriate. 
Table 3

Schedule of enrolment, intervention and data collection

\section{STUDY PERIOD}

\begin{tabular}{|c|c|c|c|c|c|}
\hline & \multirow{3}{*}{$\begin{array}{l}\text { Enrolment } \\
\text { Screening }\end{array}$} & \multirow[t]{3}{*}{ Allocation } & \multicolumn{3}{|c|}{ Post-allocation } \\
\hline & & & $\begin{array}{l}\text { Pre- } \\
\text { test }\end{array}$ & $\begin{array}{l}\text { Post- } \\
\text { test }\end{array}$ & $\begin{array}{l}\text { Follow- } \\
\text { up }\end{array}$ \\
\hline & & & Day 1 & $\begin{array}{l}\text { Week } \\
4\end{array}$ & Week 8 \\
\hline TIMEPOINT & $-t_{1}$ & 0 & $t_{1}$ & $t_{2}$ & $t_{3}$ \\
\hline \multicolumn{6}{|l|}{ ENROLMENT } \\
\hline Eligibility screen & $x$ & & & & \\
\hline Informed consent & $x$ & & & & \\
\hline Allocation & & $x$ & & & \\
\hline \multicolumn{6}{|l|}{ INTERVENTIONS } \\
\hline \multicolumn{6}{|l|}{ Intervention A } \\
\hline \multicolumn{6}{|l|}{ Intervention B } \\
\hline \multicolumn{6}{|l|}{ Control C } \\
\hline \multicolumn{6}{|l|}{ OUTCOMES } \\
\hline \multicolumn{6}{|l|}{ (ASSESSMENTS) } \\
\hline \multicolumn{6}{|l|}{ Baseline variables } \\
\hline Demographics & $x$ & & $x$ & & \\
\hline Clinical characteristics & $x$ & & $x$ & & \\
\hline \multicolumn{6}{|l|}{ Modified Rankin Scale } \\
\hline \multicolumn{6}{|l|}{ Primary outcomes } \\
\hline \multicolumn{6}{|l|}{ Feasibility } \\
\hline Recruitment rate & & & $x$ & $x$ & $x$ \\
\hline Retention rate & & & & & $x$ \\
\hline Adherence rate & & & $x$ & $x$ & $x$ \\
\hline Adverse events and falls & & & & $x$ & $x$ \\
\hline
\end{tabular}

Mini BESTest Mini Balance Evaluation Systems Test, DF dorsiflexion, PF plantarflexion 


\section{STUDY PERIOD}

Compliance with the home exercise programme

Acceptability of the intervention $\mathrm{X}$

(Individual semi-structured interviews)

Key secondary outcome

Dynamic balance $x \quad x \quad x$

(Mini BESTest)

Other secondary outcomes

Walking speed

$x \quad x \quad x$

(10-Metre Walk Test)

Functional mobility

$x$

$\mathrm{X}$

$\mathrm{X}$

(Timed Up and Go, TUG)

Dual-task functional mobility

x $x \quad x$

( $\left.T U G_{\text {manual, }} T U G_{\text {cognitive }}\right)$

Passive ankle mobility

$x \quad x \quad x$

(DF and PF range of motion measures)

Active ankle mobility

$x$

$\mathrm{X}$

$\mathrm{X}$

(DF and PF range of motion measures)

Weightbearing ankle mobility

x $\quad x \quad x$

(DF range of motion measures)

Disability \& health-related quality of life

$x \quad x \quad x$

(Stroke Impact Scale, version 2.0

Mini BESTest Mini Balance Evaluation Systems Test, DF dorsiflexion, PF plantarflexion

\section{Primary outcome}

Feasibility

a. Recruitment, retention and adherence rates 
The feasibility of conducting a full-scale RCT will be explored using predefined criteria, recording monthly recruitment and adherence rates to the face-to-face intervention. Using an exercise diary, adherence to the home exercise programme and any non-adherence will be recorded, including reasons (e.g., discontinuation due to lack of interest or injury). Any non-retention will also be recorded with reasons (e.g., consent withdrawn) (Fig. 1).

The criteria for feasibility success will be:

a. a target recruitment rate of $25 \%$ of 145 eligible patients (or 6 participants per month). The number of 145 was estimated according to information received from neurologists and clinicians practicing within $30 \mathrm{~km}$ of the study centre, concerning the number of eligible chronic stroke patients they have treated within the previous 9 months,

b. a target retention rate of $85 \%$,

c. a target minimum adherence rate of $75 \%$ for face-to-face interventions ( 6 practice sessions per week out of a maximum of 8 ) and

d. a target minimum adherence rate of $75 \%$ for home-based interventions (12 practice sessions per week out of a maximum of 16).

\section{b. Compliance with the home exercise programme}

An exercise diary will be used to report compliance. Participants in groups A and B will be asked to complete an exercise diary recording type order and repetitions performed of chosen exercises. They should also explain any problems or deviations from the home exercise protocol.

\section{c. Adverse events and falls}

Falls are considered a relevant outcome from a mobility and balance intervention study because of the associated injury risk. Any adverse events and side effects will be systematically recorded and evaluated using a formal log including the type of adverse event, dates, severity, causality differentiated into serious/non-serious, actions to restore or improve the patient's wellbeing and outcome of the event. A falls $\log$ will be provided to participants, which will be collected at $t_{2}$ and $t_{3}$, in addition to the number of and reasons for falls within prior 6 months. Study related adverse events will be treated and paid for. Severe adverse events will lead to early study termination.

\section{d. Acceptability of the intervention}

Acceptability of the intervention will be assessed using semi-structured qualitative interviews comprising four predefined questions presented in Table 2, utilised to learn how the interventions worked for the participants and how well they were appreciated. This is of clinical relevance because high acceptability may help increase motivation, autonomy and adherence in participants. 
Table 2

Semi-structured interview guide

\section{Introduction}

Main part

Question \#1 Would you please describe your experiences with the training in this study?

Question \#2 Is there anything that would motivate you to further participate in this training (i.e., after the study)?

Question \#3 What do you think about continuing with the home-exercise programme?

Question \#4 (Gp How did you experience the manual therapy of your foot? A)

Question \#4 (Gp How was the duration of the training for you?

B)

Prompt \#1 Why so?

Prompt \#2 Could you describe this in more detail?

Thanks \& Many thanks for participating in this interview.

Conclusion

Adapted from (59) and (60).

\section{Key secondary outcome}

\section{Dynamic balance (Mini Balance Evaluation Systems Test, Mini BESTest)}

The primary outcome will be dynamic balance as assessed by the Mini Balance Evaluation Systems Test (BESTest) (25). Fourteen items are rated on a 3 -point ordinal scale (from $0=$ severe to $2=$ normal). A transformation table from raw into interval scores is available since the Mini BESTest demonstrated excellent construct validity, unidimensionality, minimal ceiling and no floor effects (25) and a minimal detectable change of 3.0 points (26). The 4 subscales are transitions/anticipatory postural control, reactive postural control, sensory orientation and stability in gait. Preliminary changes in the key secondary outcome will be used to calculate the sample size for the main trial.

\section{Other secondary outcomes}

Participants may use their walking aid for all walking tests, but this needs to be documented and used consistently for all trials.

\section{a. Walking speed (10-Metre Walk Test, 10MWT)}

The 10MWT at maximum speed will be used as a functional measure of walking. It has been recommended by various task forces $(27,28)$. Academy of Neurologic Physical Therapy test instructions will be followed and data recorded in $\mathrm{m} / \mathrm{s}$. The average will be calculated from two trials. 
For the 10MWT, an excellent concurrent validity has been shown with ADL (29). Good convergent validity with the 6-Minute Walk Test (30) has been observed, an excellent interrater and test-retest reliability and a minimum detectable change (MDC) of 16\% (31).

\section{b. Functional mobility (Timed Up and Go, TUG)}

The TUG (32) is a measure of functional mobility able to detect change over time in patients post stroke (31). The participant is observed and timed while he or she rises from a chair with armrests, walks 3 metres as quickly and safely as possible towards a marking, turns, walks back and sits. Excellent interrater reliability has been found, plus good convergent validity with relevant measures (32). The MDC of the TUG in stroke patients is $23 \%$ (31).

\section{c. Functional mobility with dual tasking (TUG manual $_{\text {and }} T U_{\text {cognitive }}$ )}

Dual tasking will be assessed using the $T U G_{\text {manual }}$ and $T U G_{\text {cognitive }}$ where the TUG is combined with a motor (carrying) or cognitive task (subtracting in threes) (33). For both dual-tasking TUGs, a good testretest reliability and convergent validity have been confirmed $(34,35)$. An MDC of 3.53 seconds for the TUG manual (34) has been observed in people after stroke. The time difference between the TUG and

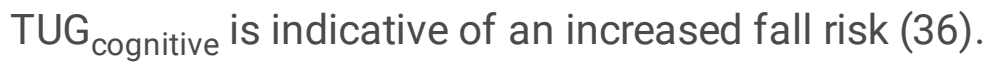

\section{d. Ankle range of motion (passive and active dorsiflexion and plantarflexion ROM)}

Ankle mobility will be assessed using different techniques to assess the effects of MT.

\section{Non-weightbearing dorsiflexion and plantarflexion ROM measurement}

Passive and active talocrural dorsiflexion (DF) and plantarflexion (PF) ROM will be assessed in nonweight-bearing supine position with the knees on a standard knee roll ( $20^{\circ}$ flexed), to avoid tensioning of the gastrocnemius muscle (37). A standard measurement technique is described in detail in Additional File 3. Data will be recorded using the neutral-zero method (38). Excellent interrater reliability has been demonstrated for ankle dorsiflexion (37) and plantarflexion measures (39).

\section{Weightbearing dorsiflexion ROM measurement}

Ankle dorsiflexion ROM will also be assessed utilising a tape measure and a weight-bearing lunge facing a wall, as validated by Konor et al. (2012) and outlined in detail in Additional File 3 (40). Excellent interrater (41) and intrarater reliability, low measurement error and an MDC of $1.1-1.5 \mathrm{~cm}$ has been demonstrated for this measurement technique (40).

\section{e. Disability and HRQoL after stroke (Stroke Impact Scale, version 2.0, SIS v2.0)}

The validated German version (42) of the SIS v2.0 (43) will be used to assess disability and HRQoL. The SIS is a stroke-specific PROM comprising 64 items and assessing 8 domains (strength, memory, emotion, communication, ADL and instrumented ADL (IADL), mobility, hand function, and social participation). A summary score is not calculated, but a physical domain score can be created from the strength, hand function, mobility and ADL/IADL domains. In the emotion domain, items 3a-e and $3 \mathrm{~g}$ are reverse scored, 
with all items scored on a 5-point Likert scale. Scores for each domain are transformed to a 0-100 score with lower scores representing poor HRQoL; using the formula: Transformed Scale = [(Actual raw score lowest possible raw score) / Possible raw score range] * 100. In addition, the SIS v2.0 includes a 0-100 visual analogue scale, with 0 representing no recovery and 100 representing full recovery.

Moderate to excellent internal consistency, test-retest reliability, convergent, divergent and discriminant validity have been demonstrated for the SISv2 (43).

\section{Participant timeline}

The planned study duration is from 11.9.2020 to 31.5.2021. A detailed overview of the participant timeline is shown in Table 3.

\section{Sample size}

The study will be a pilot RCT to provide information for a subsequent larger RCT. The sample size was based on specific objectives of the study such as feasibility, acceptability and falls rather than on estimated effects of interventions. Julious recommends a minimum sample size of 12 participants per group for pilot studies (44). Therefore, 36 people post stroke will be recruited into three groups (A, $B$ and C) For the sample size estimation, imprecision of the standard deviation in small samples will be taken into account (45).

\section{Data management and data protection}

Data quality will be promoted by duplicate (10MWT) or triple measurements (ankle ROM measures, utilising two different measurement approaches). Only the PI and care provider will be aware of the demographic data and have access to the pseudonymisation documents. Double data entry will be performed. Data will be verified by conducting spot checks of value ranges and field types for certain data fields, and logical checks will also be performed (e.g. the order of visits). When checking value ranges, the fields will be checked for permitted values. Field type checks concern whether the values entered fit the field definition (e.g. numerical fields). Any discrepancies found will be rectified.

For the qualitative analysis of interviews, coding will be performed by two independent coders (KT, BS). Codes will be discussed and if a consensus cannot be reached, a third researcher will provide support (FS). Post interview, the interviewer will review and ascertain that all answers have been understood correctly. Participants will be asked to provide feedback on the findings. For the report, participant quotations will be used, together with participant ID and group allocation.

German legislation states any study records and documents must be stored for a period of 10 years after the conclusion of the clinical study. Personal data will be collected, forwarded, stored and evaluated as part of this clinical study in accordance with the legal provisions of the General Data Protection Regulation (EU) 2016/679 (GDPR) and German Data Protection Act 2018 (BDSG).

\section{Data analyses}

Statistical analysis 
IBM SPSS Software, release 26.0 (IBM Corporation, Armonk, NY, USA) and GraphPad Prism 8, San Diego, California, will be used for the data analyses. Statistical significance will be defined as a two-tailed pvalue $<0.05$. The percentage of missing data will be recorded. Using Little's test of missing completely at random (MCAR) (46) the data will be checked, signified by a p-value $>0.05$. An intention-to-treat analysis will be performed.

Descriptive statistics will be used for the baseline demographic variables, primary and secondary outcomes. Continuous data will be checked for normal distribution using the Shapiro Wilk Test, Q-Q plots and histograms. Raw count (number $(\mathrm{N})$, \%) will be presented for counted ( $\mathrm{N}$ females and males, $\mathrm{N}$ falls and $\mathrm{N}$ adverse events if any, compliance (types and order of home exercises, $\mathrm{N}$ of repetitions and deviations from the protocol) and nominal data (gender, recruitment, retention and adherence rates). Medians (range; 25th and 75th percentiles) will be reported for ordinal data (Mini BESTest if there are missing data, SIS), mean (95\% confidence interval (CI)) will be reported for continuous data (age, Mini BESTest if data can be transformed into interval level, 10MWT, TUG, TUG manual, TUG $_{\text {cognitive, }}$ ankle ROM measures).

The recruitment rate (\%) will be estimated by dividing the number of consenting participants by the $\mathrm{N}$ of eligible patients, multiplied by 100 . The retention rate determined by dividing the $\mathrm{N}$ of participants who completed the study by the $\mathrm{N}$ of the total sample, times 100 . Adherence rates will be calculated by dividing the $\mathrm{N}$ of TOT sessions $(8 \mathrm{x} ; 16 \mathrm{x})$ performed by the participants, divided by the $\mathrm{N}$ of the scheduled (face-to-face; home-based) training sessions over the 4 week study period, times 100 (47). The eligibility, recruitment and adherence rates will be calculated with their $95 \% \mathrm{Cl}$ according to the Wilson 'score' method cited by Newcombe (48); when the proportion is close to 0 or 1, a Poisson approximation as described by Brown and colleagues will be used (49).

Inductive statistics will detect any trends related to the intervention efficacy acting as a basis for the sample size calculation for a larger main study. The sample size estimation will be based on a power of $80 \%$, an alpha of $5 \%$ and the standardised effect size (Cohen's d). Cohen's $d$ will be calculated using the formula $d=($ mean $1-$ mean 2)/corrected pooled SD. The corrected pooled SD for $80 \%$ of trials will be determined using the formula (square root from ((SD12 + SD22)/2))*1.293 (45). Effect sizes will be estimated with $95 \% \mathrm{Cl}$ using an effect size calculator provided by the Centre for Evaluation and Monitoring at the University of Durham, UK.

Baseline group differences will be examined using Chi-square test for nominal data, Kruskal Wallis test for ordinal data and one-way analysis of variance (ANOVA) for continuous data. If a non-normal data distribution is observed in continuous data, data transformation or non-parametrical tests will be performed, as appropriate. Kruskal Wallis test will evaluate differences between groups and timepoints, followed by a Bonferroni correction for multiple comparisons. On continuous data, test assumptions for a mixed design ANOVA will be reviewed, with standard correction procedures employed appropriately. In order to evaluate the effects of the intervention on walking speed, mobility, dual-task mobility and ankle ROM, a 2-way mixed design ANOVA will be used, with "time" $\left(t_{1}, t_{2}\right.$ and $\left.t_{3}\right)$ as within-subject factor and 
"group" (A, B and C) as between-subject factor. If the overall group-by-time interaction is statistically significant, post-hoc tests with Bonferroni correction will be applied to determine group differences in pairwise comparisons.

\section{Qualitative data analysis}

Interviews will be transcribed applying the Dresing and Pehl (2017) transcription rules (50) using F4 Transkript software (Dr. Dresing \& Pehl GmbH, Marburg, Germany). Data analysis will be conducted with MaxQDA software (VERBI GmbH, Berlin, Germany) adhering to the Consolidated Criteria for Reporting Qualitative Research (COREQ) (51). Qualitative content analysis will use a systematic and consistent approach to enhance rigour (52-54).

Coding of the total dataset will be performed by two independent researchers to increase intersubjective conformability (55). Recurring ideas, concepts, themes and words will be identified. A combined inductive and deductive category development approach, based on the material and the research question will be used. A coding frame where categories are required to be (apparently) unidimensional, mutually exclusive and exhaustive will be developed. Each dimension should capture a different concept of the text.

Relevant material will be selected, structured and marked, text sections segmented to identify main and subcategories. Categories will be defined, named and characterised. Subcategory saturation indicated after one use. A minimum length of a segment is one sentence. Categories will be continuously adapted (54). A data matrix will be created and descriptive statistics performed e.g., frequencies. Categories and subcategories will be illustrated using quotations together with a participant ID and group number ( $A, B$ or C). Credibility will be further enhanced through a reflective diary for documenting decisions (reflexivity) and peer debriefing of the interviewer to identify biases and assumptions (truth value) (55). Records will be kept carefully to ensure consistent and transparent data interpretation (56). Comparisons across accounts will be sought to ensure various perspectives are represented (57).

\section{Dissemination}

Study results will be disseminated at national and international conferences and published in peerreviewed journals. Study participants will be informed about the study findings via lay-language letters.

\section{Discussion}

Walking impairment and falls lead to reduced social participation in many people post stroke. Patients experience limitations in body structure, function, activities and participation as classified by the World Health Organisation (58), and consequences are manifold. Therefore, we considered a combined intervention appropriate, addressing functional, structural and activity components. The aim will be to evaluate the feasibility of a larger trial and explore the preliminary effects of a specific mobility and balance TOT versus its combination with ankle MT on balance, mobility and falls in people post stroke. Study results will contribute to the stroke rehabilitation literature as the intervention combines traditional MT approaches with specific TOT using a systematic approach. A goal-oriented training will be employed encouraging external focus. Predefined progression criteria will be used to enable a targeted training 
close to an individual's physical performance limits. By using ADL-based tasks we hope to induce further performance gains in study participants, all in the chronic stage after stroke. These tasks could be of some relevance to the participants, with a likely transfer into their daily lives occurring. Designed for an outpatient setting in a rural area of Germany, the intervention is regarded practicable, low-cost in terms of equipment and readily transferable to other activities. Feasibility and effectiveness provided this may facilitate the implementation of the novel treatment also in outpatient rural environments. Study findings could be applied to devise research projects in people with other neurological disorders involving the upper motor neuron, such as multiple sclerosis or traumatic brain injury.

\section{Study limitations}

We acknowledge that this feasibility study has a small sample size, designed to evaluate the interventions' preliminary effectiveness, in order to calculate the main study sample size. All results are to be considered preliminary and need to be validated by a larger study. In this study setting, the lack of objective outcome instruments e.g., motion capture systems, posturography or digital pressure sensing platforms may be limiting, Notwithstanding, all ROM measurements are based on relevant literature and are clearly described in the study protocol. Standardised instructions of the two assessors will be provided to enhance inter-rater reliability. In addition, validated tests and questionnaires will be used representing gold standard measurements post stroke. Actual falls will be recorded throughout the study and compared against the patient-reported fall rate within the 6 months prior. It is recognised that some participants may not correctly recall their fall rate, but generally, patients consider falls relevant and may therefore remember them. A strength of this study appears to be the follow-up assessment. It will allow evaluation of longer-term changes following TOT with and without MT. A control group receiving usual care will be used to compare changes. This study is planned to be conducted as a single-centre study, the follow-up main RCT could try to recruit several centres to achieve a representative sample according to the sample calculation, to allow for a generalisation of the results.

\section{Trial Status}

The protocol published here is version 1.3 dated 02.08.2020. The trial began recruitment on 25.09.2020 and is expected to continue until 31.05.2021.

\section{Abbreviations}

ADL: activities of daily living; ANOVA: analysis of variance; AP: anterior-posterior; BDSG: German Data Protection Act; Mini BESTest: Balance Evaluation Systems Test, short version; Cl: confidence interval; CONSORT: Consolidated Standards of Reporting Statement; COREQ: Consolidated Criteria for Reporting Qualitative Research; CRF: case report form; DF: dorsiflexion: $\eta^{2}$ : partial Eta-squared; GDPR: General Data Protection Regulation; Gp: group; HRQoL: health-related quality of life; IADL: instrumented activities of daily living; ID: identification number; MCAR: missing completely at random; MDC: minimum detectable change; mRS: Modified Rankin Scale; MT: manual therapy; N: number; PA: posterior-anterior; PF: 
plantarflexion; PI: principle investigator; RCT: randomised controlled trial; ROM: range of motion; SD: standard deviation; SIS v2.0: Stroke Impact Scale, version 2.0; t: test; TOT: task-oriented training; TUG:

Timed Up and Go; TUG $_{\text {cognitive: }}$ TUG with cognitive dual tasking; $\mathbf{T U G}_{\text {manual }}$ : TUG with motor dual tasking; 10MWT: 10-Metre Walk Test.

\section{Declarations}

\section{Ethics approval and consent to participate}

Ethics approval was received on the 10.9.2020 from the responsible ethics committee of the Bavarian State Medical Association (Bayerische Landesärztekammer), reference number 20065. Written informed consent will be obtained from all participants.

\section{Consent for publication}

Not applicable. No details, images, or videos relating to an individual person will be published, all data will be presented in aggregate.

\section{Availability of data and materials}

Datasets generated and/or analysed during the current study will be available from the corresponding author on reasonable request.

\section{Competing interests}

The authors declare no competing interests.

\section{Funding}

This study is self-funded by the initiating investigator (KT) in the course of her Master's studies in neurological physiotherapy at the Department for Health Sciences, Medicine and Research, Faculty of Health and Medicine, Danube University Krems, Austria. VASCage $\mathrm{GmbH}$, Research Centre on Vascular Ageing and Stroke, Innsbruck, Austria supports the indemnity insurance costs and has no role in the design of the study and collection, analysis, and interpretation of data.

\section{Authors' contributions}

FS is the PI of the study; KT and BS conceived the study, developed the study protocol and applied for funding and ethical approval. KT delivers the intervention, EB and EK contributed to the study design and the development of the protocol; they are the study assessors. BS is the academic supervisor of KT, drafted the manuscript and is responsible for the data analysis. All authors read and approved the final manuscript. 
The authors acknowledge the contribution of Mr. Josef Maurer with provision of rehabilitation facilities; and support from Prof. Michaela Pinter and Mag. Martina Tüchler from the Department for Health Sciences, Medicine and Research, Faculty of Health and Medicine, Danube University Krems, Austria.

\section{References}

1. van de Port IG, Kwakkel G, van Wijk I, Lindeman E. Susceptibility to deterioration of mobility longterm after stroke: a prospective cohort study. Stroke. 2006;37(1):167-71.

2. Bower K, Thilarajah S, Pua YH, Williams G, Tan D, Mentiplay B, et al. Dynamic balance and instrumented gait variables are independent predictors of falls following stroke. Journal of neuroengineering and rehabilitation. 2019;16(1):3.

3. Aho K, Harmsen P, Hatano S, Marquardsen J, Smirnov VE, Strasser T. Cerebrovascular disease in the community: results of a WHO collaborative study. Bulletin of the World Health Organization. 1980;58(1):113-30.

4. Jakubowitz E, Yao D, Windhagen $H$, Stukenborg-Colsman $C$, Thomann A, Daniilidis K. [Treatment Options for Neurogenic Drop Foot: A Systematic Literature Research]. Zeitschrift fur Orthopadie und Unfallchirurgie. 2017;155(4):402-8.

5. Given JD, Dewald JP, Rymer WZ. Joint dependent passive stiffness in paretic and contralateral limbs of spastic patients with hemiparetic stroke. Journal of neurology, neurosurgery, and psychiatry. 1995;59(3):271-9.

6. Vér $C$, Emri M, Spisák T, Berényi E, Kovács K, Katona P, et al. The Effect of Passive Movement for Paretic Ankle-Foot and Brain Activity in Post-Stroke Patients. Eur Neurol. 2016;76(3-4):132-42.

7. Kim SL, Lee BH. The Effects of Posterior Talar Glide and Dorsiflexion of the Ankle Plus Mobilization with Movement on Balance and Gait Function in Patient with Chronic Stroke: A Randomized Controlled Trial. Journal of neurosciences in rural practice. 2018;9(1):61-7.

8. Park D, Lee JH, Kang TW, Cynn HS. Four-week training involving ankle mobilization with movement versus static muscle stretching in patients with chronic stroke: a randomized controlled trial. Topics in stroke rehabilitation. 2019;26(2):81-6.

9. Kluding PM, Santos M. Effects of ankle joint mobilizations in adults poststroke: a pilot study. Arch Phys Med Rehabil. 2008;89(3):449-56.

10. An CM, Jo SO. Effects of Talocrural Mobilization with Movement on Ankle Strength, Mobility, and Weight-Bearing Ability in Hemiplegic Patients with Chronic Stroke: A Randomized Controlled Trial. J Stroke Cerebrovasc Dis. 2017;26(1):169-76.

11. Shumway-Cook A, Woollacott MH. Motor control: translating research into clinical practice. $5^{\text {th }}$ ed ed. Market Street, Philadelphia: Wolters Kluver; 2017.

12. Rensink $M$, Schuurmans $M$, Lindeman $E$, Hafsteinsdóttir T. Task-oriented training in rehabilitation after stroke: systematic review. Journal of advanced nursing. 2009;65(4):737-54. 
13. Wevers L, van de Port I, Vermue M, Mead G, Kwakkel G. Effects of task-oriented circuit class training on walking competency after stroke: a systematic review. Stroke; a journal of cerebral circulation. 2009;40(7):2450-9.

14. Veerbeek JM, van Wegen E, van Peppen R, van der Wees PJ, Hendriks E, Rietberg M, et al. What is the evidence for physical therapy poststroke? A systematic review and meta-analysis. PloS one. 2014;9(2):e87987.

15. Carr J, Shepherd R. Neurological rehabilitation. $2^{\text {nd }}$ ed ed. London: Churchill Livingstone Elsevier; 2010.

16. Winstein CJ, Kay DB. Translating the science into practice: shaping rehabilitation practice to enhance recovery after brain damage. Progress in brain research. 2015;218:331-60.

17. Bauder $\mathrm{H}$, Taub E, Miltner W. Behandlung motorischer Störungen nach Schlaganfall: Die Taubsche Bewegungsindukationstherapie. Göttingen: Hogrefe; 2001.

18. French B, Thomas LH, Coupe J, McMahon NE, Connell L, Harrison J, et al. Repetitive task training for improving functional ability after stroke. The Cochrane database of systematic reviews. 2016;11(11):Cd006073.

19. Wulf G, Lewthwaite R. Optimizing performance through intrinsic motivation and attention for learning: The OPTIMAL theory of motor learning. Psychonomic bulletin \& review. 2016;23(5):1382414.

20. Sabbah A, El Mously S, Elgendy HHM, Farag MAAE, Elwishy AAB. Functional outcome of joint mobilization added to task-oriented training on hand function in chronic stroke patients. The Egyptian Journal of Neurology, Psychiatry and Neurosurgery. 2020;56(1):38.

21. van Swieten JC, Koudstaal PJ, Visser MC, Schouten HJ, van Gijn J. Interobserver agreement for the assessment of handicap in stroke patients. Stroke; a journal of cerebral circulation. 1988;19(5):6047.

22. Mintken PE, Derosa C, Little T, Smith B. A Model for Standardizing Manipulation Terminology in Physical Therapy Practice. The Journal of orthopaedic and sports physical therapy. 2008;38(3):A1A6.

23. Hoffmann TC, Glasziou PP, Boutron I, Milne R, Perera R, Moher D, et al. Better reporting of interventions: template for intervention description and replication (TIDieR) checklist and guide. BMJ : British Medical Journal. 2014;348:g1687.

24. Peek K, Sanson-Fisher R, Mackenzie L, Carey M. Interventions to aid patient adherence to physiotherapist prescribed self-management strategies: a systematic review. Physiotherapy. 2016;102(2):127-35.

25. Franchignoni F, Horak F, Godi M, Nardone A, Giordano A. Using psychometric techniques to improve the Balance Evaluation Systems Test: the mini-BESTest. Journal of rehabilitation medicine : official journal of the UEMS European Board of Physical and Rehabilitation Medicine. 2010;42(4):323-31.

26. Tsang CS, Liao LR, Chung RC, Pang MY. Psychometric properties of the Mini-Balance Evaluation Systems Test (Mini-BESTest) in community-dwelling individuals with chronic stroke. Phys Ther. 
2013;93(8):1102-15.

27. Hebert D, Lindsay MP, Mclntyre A, Kirton A, Rumney PG, Bagg S, et al. Canadian stroke best practice recommendations: Stroke rehabilitation practice guidelines, update 2015. Int J Stroke. 2016;11(4):459-84.

28. Sullivan JE, Crowner BE, Kluding PM, Nichols D, Rose DK, Yoshida R, et al. Outcome measures for individuals with stroke: process and recommendations from the American Physical Therapy Association neurology section task force. Phys Ther. 2013;93(10):1383-96.

29. Maeda A, Yuasa T, Nakamura K, Higuchi S, Motohashi Y. Physical performance tests after stroke: reliability and validity. Am J Phys Med Rehabil. 2000;79(6):519-25.

30. Donovan K, Lord SE, McNaughton HK, Weatherall M. Mobility beyond the clinic: the effect of environment on gait and its measurement in community-ambulant stroke survivors. Clin Rehabil. 2008;22(6):556-63.

31. Flansbjer UB, Holmbäck AM, Downham D, Patten C, Lexell J. Reliability of gait performance tests in men and women with hemiparesis after stroke. J Rehabil Med. 2005;37(2):75-82.

32. Podsiadlo D, Richardson S. The timed "Up \& Go": a test of basic functional mobility for frail elderly persons. Journal of the American Geriatrics Society. 1991;39(2):142-8.

33. Shumway-Cook A, Brauer S, Woollacott M. Predicting the probability for falls in community-dwelling older adults using the Timed Up \& Go Test. Phys Ther. 2000;80(9):896-903.

34. Chan PP, Si Tou JI, Tse MM, Ng SS. Reliability and Validity of the Timed Up and Go Test With a Motor Task in People With Chronic Stroke. Arch Phys Med Rehabil. 2017;98(11):2213-20.

35. Hofheinz M, Schusterschitz C. Dual task interference in estimating the risk of falls and measuring change: a comparative, psychometric study of four measurements. Clin Rehabil. 2010;24(9):831-42.

36. Pohl PS, Gras LZ, Bosch PR, Ganley KJ, Mayer J. Dual Task Timed Up-and-Go for Older Adults With and Without Balance Deficits. Physical \& Occupational Therapy In Geriatrics. 2019;37(4):247-59.

37. Baumbach SF, Brumann M, Binder J, Mutschler W, Regauer M, Polzer H. The influence of knee position on ankle dorsiflexion - a biometric study. BMC musculoskeletal disorders. 2014;15:246.

38. Debrunner HU. Gelenkmessung (Neutral-0-Methode), Längenmessung, Umfangmessung. Bern: Bulletin des Offiziellen Organs der Arbeitsgemeinschaft für Osteosynthesefragen; 1971.

39. Alawna MA, Unver BH, Yuksel EO. The Reliability of a Smartphone Goniometer Application Compared With a Traditional Goniometer for Measuring Ankle Joint Range of Motion. Journal of the American Podiatric Medical Association. 2019;109(1):22-9.

40. Konor MM, Morton S, Eckerson JM, Grindstaff TL. Reliability of three measures of ankle dorsiflexion range of motion. International journal of sports physical therapy. 2012;7(3):279-87.

41. Bennell KL, Talbot RC, Wajswelner H, Techovanich W, Kelly DH, Hall AJ. Intra-rater and inter-rater reliability of a weight-bearing lunge measure of ankle dorsiflexion. The Australian journal of physiotherapy. 1998;44(3):175-80. 
42. Petersen C, Morfeld M, Bullinger M. [Testing and validation of the German version of the Stroke Impact Scale]. Fortschritte der Neurologie-Psychiatrie. 2001;69(6):284-90.

43. Duncan PW, Wallace D, Lai SM, Johnson D, Embretson S, Laster LJ. The stroke impact scale version 2.0. Evaluation of reliability, validity, and sensitivity to change. Stroke; a journal of cerebral circulation. 1999;30(10):2131-40.

44. Julious SA. Sample size of 12 per group rule of thumb for a pilot study. Pharmaceutical statistics. 2005;4:287-91.

45. Vickers AJ. Underpowering in randomized trials reporting a sample size calculation. Journal of clinical epidemiology. 2003;56(8):717-20.

46. Little RJA. A Test of Missing Completely at Random for Multivariate Data with Missing Values. Journal of the American Statistical Association. 1988;83(404):1198-202.

47. Osterberg L, Blaschke T. Adherence to medication. The New England journal of medicine. 2005;353(5):487-97.

48. Newcombe RG. Two-sided confidence intervals for the single proportion: comparison of seven methods. Statistics in medicine. 1998;17(8):857-72.

49. Brown L, Cai T, DasGupta A. Interval estimation for a binomial proportion. Statistical Science. 2001;16(2):101-33.

50. Praxisbuch Interview, Transkription \& Analyse. Anleitungen und Regelsysteme für qualitativ Forschende. 8. ed. Marburg: Eigenverlag; 2018.

51. Tong A, Sainsbury P, Craig J. Consolidated criteria for reporting qualitative research (COREQ): a 32item checklist for interviews and focus groups. International Journal for Quality in Health Care. 2007;19(6):349-57.

52. Berelson B. Content Analysis in Communication Research. Glencoe: Free Press; 1952.

53. Mayring P. Qualitative Inhaltsanalyse. Grundlagen und Techniken. Weinheim, Deutschland: Beltz; 2003.

54. Steigleder S. Die strukturierende qualitative Inhaltsanalyse im Praxistest: eine konstruktiv kritische Studie zur Auswertungsmethodik von Philipp Mayring: Tectum-Verlag; 2008.

55. Noble H, Smith J. Issues of validity and reliability in qualitative research. Evidence-based nursing. 2015;18(2):34-5.

56. Lincoln YS, Guba EG. Naturalistic inquiry. Beverly Hills, CA: Sage; 1985.

57. Slevin E, Sines D, editors. Enhancing the truthfulness consistency and transferability of a qualitative study 2000.

58. Organization WH. International Classification of Functioning, Disability and Health ICF Geneva, Switzerland: WHO; 2010 [updated 2014.

59. Helfferich C. Die Qualität qualitativer Daten: Manual für die Durchführung qualitativer Interviews. Wiesbaden: VS Verlag für Sozialwissenschaften. Springer Fachmedien; 2011. 
60. Olsson JE, Ekblad S, Bertilson BC, Toth-Pal E. Swedish adaptation of the General Medical Council's multisource feedback questionnaires: a qualitative study. International journal of medical education. 2018;9:161-9.

\section{Figures}

\section{CONSORT Flow Diagram (Extension to randomised pilot and feasibility trials; Eldridge et al., 2016)}

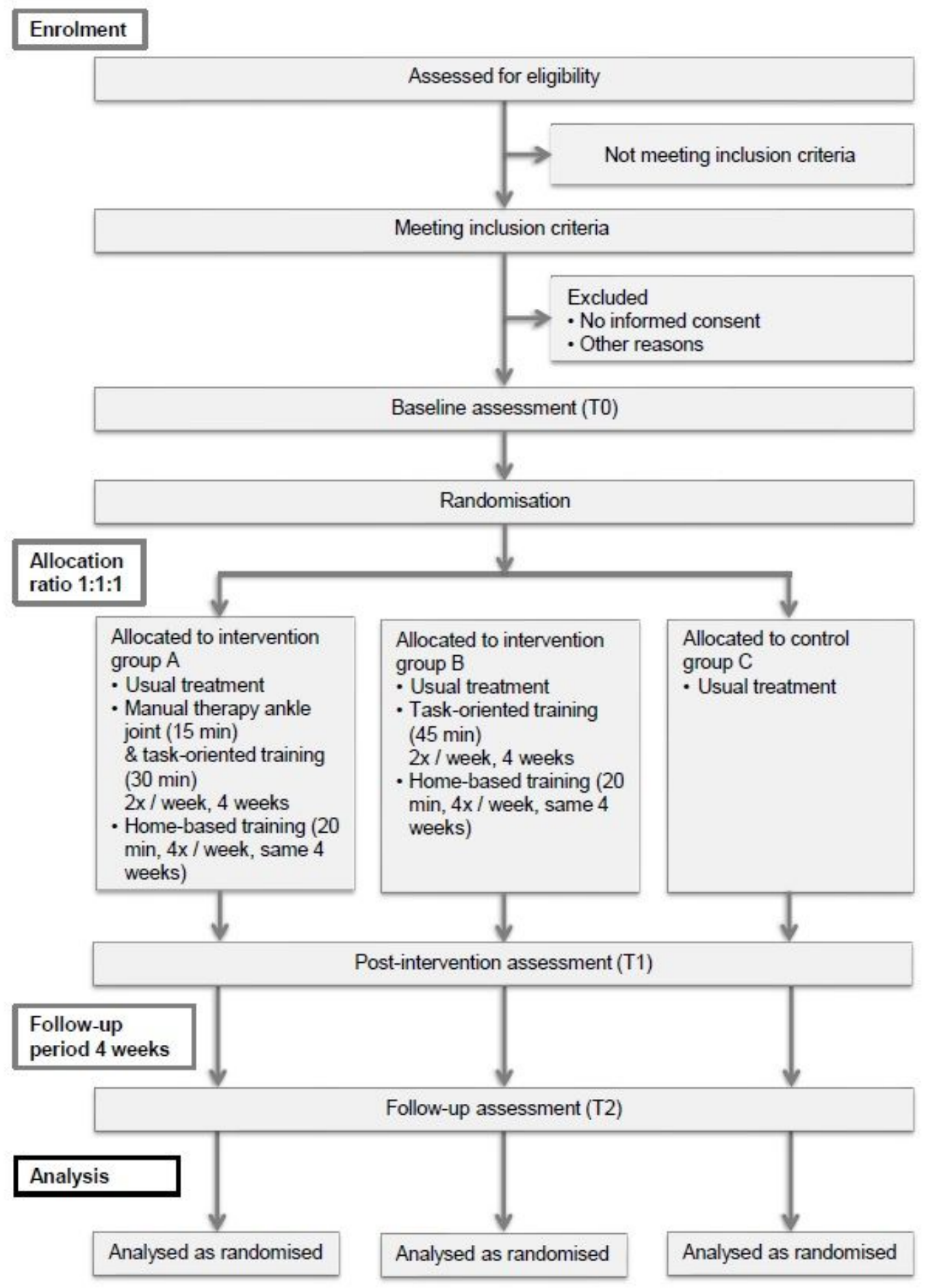




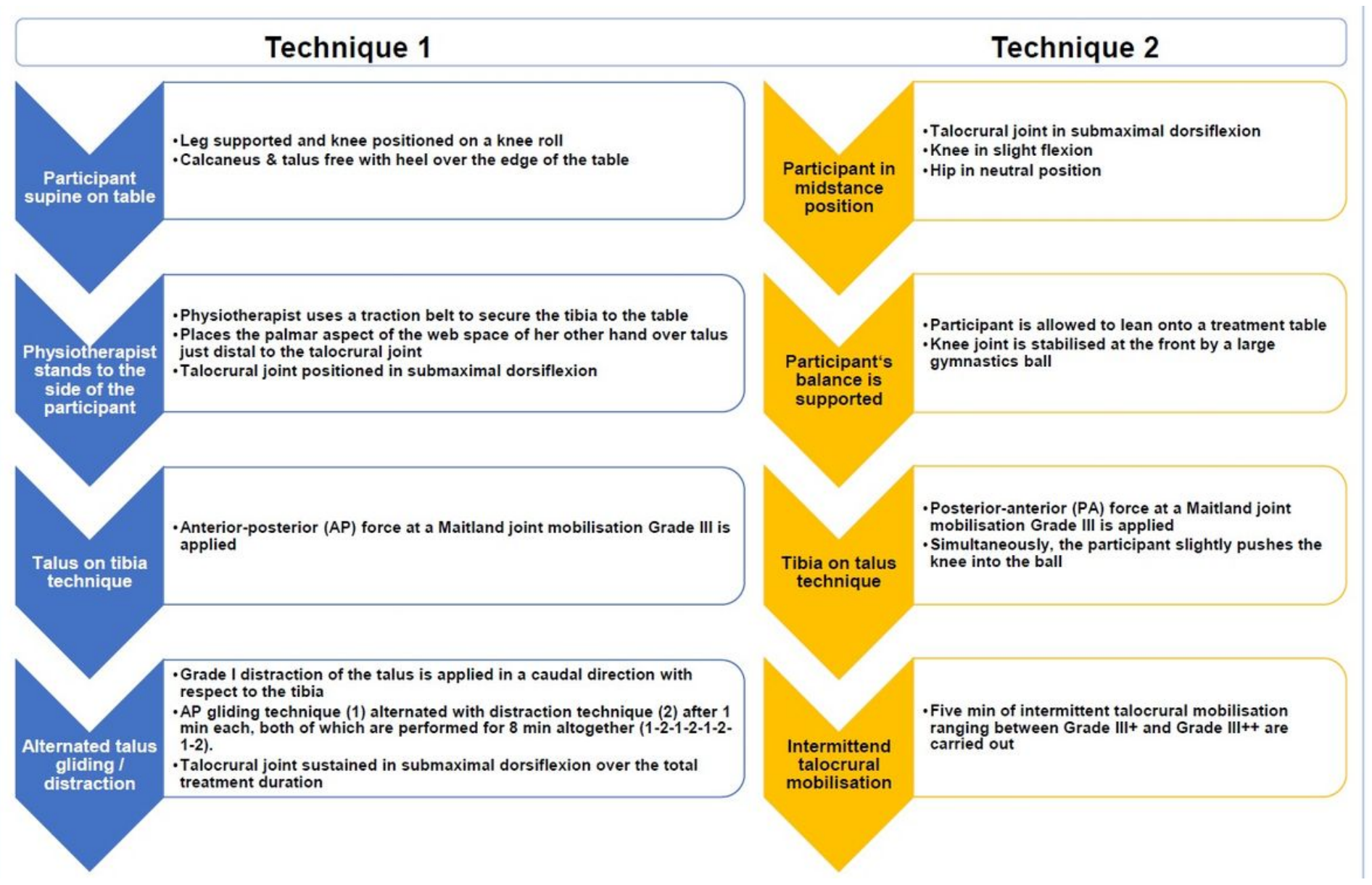

\section{Figure 2}

Manual therapy techniques

\section{Supplementary Files}

This is a list of supplementary files associated with this preprint. Click to download.

- Additionalfile1CONSORTpilotstudies.pdf

- Additionalfile2Intervention.pdf

- Additionalfile3Measurement.pdf 